\title{
The effects of Bu Yang Huan Wu Tang on post- stroke epilepsy: a nationwide matched study
}

This article was published in the following Dove Press journal:

Clinical Epidemiology

\author{
Shu-Wen Wengl,* \\ Ta-Liang Chen ${ }^{2-4}$ \\ Chun-Chieh Yeh ${ }^{5,6}$ \\ Hsin-Long Lane ${ }^{7}$ \\ Chien-Chang Liao ${ }^{2-4,8, *}$ \\ Chun-Chuan Shih ${ }^{7,9}$ \\ 'Department of Chinese Medicine, \\ Taichung Hospital, Ministry of \\ Health and Welfare, Taichung, Taiwan; \\ ${ }^{2}$ Department of Anesthesiology, \\ Taipei Medical University Hospital, \\ Taipei, Taiwan; ${ }^{3}$ Anesthesiology and \\ Health Policy Research Center, \\ Taipei Medical University Hospital, \\ Taipei, Taiwan; ${ }^{4}$ Department of \\ Anesthesiology, School of Medicine, \\ College of Medicine, Taipei \\ Medical University, Taipei, Taiwan; \\ ${ }^{5}$ Department of Surgery, China \\ Medical University Hospital, Taichung, \\ Taiwan; ${ }^{6}$ Department of Surgery, \\ University of Illinois, Chicago, IL, \\ USA; ${ }^{7}$ School of Chinese Medicine \\ for Post-Baccalaureate, I-Shou \\ University, Kaohsiung, Taiwan; ${ }^{8}$ School \\ of Chinese Medicine, China Medical \\ University, Taichung, Taiwan; ${ }^{~}$ Program \\ for the Clinical Drug Discovery \\ from Botanical Herbs, Taipei Medical \\ University, Taipei, Taiwan
}

*These authors contributed equally to this work

Correspondence: Chun-Chuan Shih School of Chinese Medicine for PostBaccalaureate, I-Shou University, 8 Yida Road, Kaohsiung City 824, Taiwan Tel +8867615 I I 00 ext. 7078

Fax +8867615 5150

Email hwathai@seed.net.tw
Objective: To compare the long-term risk of epilepsy in stroke patients who use Bu Yang Huan Wu Tang (BYHWT) and those who do not.

Methods: In the Taiwanese national insurance claims data, we identified newly diagnosed stroke patients receiving inpatient care in the years 2000-2004. Using propensity score-matched pairs to balance the baseline characteristics, we selected eligible stroke patients who did $(n=8,971)$ and did not $(n=8,971)$ receive BYHWT. These two groups were followed up until the end of 2009 to track the occurrence of epilepsy. We used Cox proportional hazard models to calculate the adjusted HRs and 95\% CIs for post-stroke epilepsy during the follow-up period according to BYHWT use.

Results: Compared with the control group, stroke patients with BYHWT had a reduced risk of epilepsy during the 5-9 years of the follow-up period (HR $0.69,95 \%$ CI $0.61-0.77$ ). The association between BYHWT and reduced post-stroke epilepsy was significant in various subgroups of stroke patients. There was a dose-dependent decrease in the frequency of epilepsy with increasing quantities of BYHWT use from 1 package (HR $0.77,95 \%$ CI $0.66-0.90$ ) to $\geq 6$ packages (HR 0.52, 95\% CI 0.42-0.65).

Conclusion: Stroke patients who received BYHWT therapy had a reduced long-term risk of epilepsy, and the beneficial effect could be observed in various subgroups. However, future clinical trials will be necessary to corroborate the present findings and identify the biochemical mechanism involved.

Keywords: stroke, epilepsy, Bu Yang Huan Wu Tang, long-term risk

\section{Introduction}

Stroke is the second leading cause of death and years of life lost among adults worldwide. ${ }^{1}$ Approximately 5.5 million deaths globally were attributed to stroke in 2016 , and the annual cost of stroke was estimated to be $\sim$ US $\$ 30,000$ per patient worldwide. ${ }^{1,2}$ Along with depression, acute myocardial infarction, sleep disorders, pneumonia, gastrointestinal bleeding, urinary tract infection, recurrent stroke, and post-stroke falls, epilepsy is considered a common complication after stroke. ${ }^{3-7}$ The prevalence of epilepsy among stroke patients was estimated to range from $6.3 \%$ to $12.4 \% .{ }^{8.9}$ After the first seizure, patients may develop recurrent seizures and exhibit increased fatality. ${ }^{10}$

Traditional Chinese medicine (TCM) is commonly used in Taiwan and other Asian countries. ${ }^{11-13} \mathrm{~A}$ high rate of TCM use among stroke patients in Taiwan was reported in a previous nationwide study, ${ }^{14}$ and receiving adjuvant TCM therapy may be associated with reduced risk of adverse outcomes after stroke. ${ }^{15}$ One component in TCM is Chinese herbal medicine; Bu Yang Huan Wu Tang (BYHWT) is commonly 
prescribed Chinese herbal formula for stroke patients in Taiwan. ${ }^{16,17}$ Experimental studies have indicated that BYHWT has neuroprotective and neurogenesis-promoting effects in rats with cerebral ischemia. ${ }^{18-21}$ Huanqi is one of the components of BYHWT that was suggested as a potent prescription for the treatment of endothelium-related vascular complications. ${ }^{49}$ However, limited information is available regarding whether BYHWT has beneficial antiepileptic effects in stroke patients.

Using insurance-based data from Taiwan, we conducted a nationwide retrospective cohort study with matching by propensity score to investigate the risk of epilepsy in stroke patients who received BYHWT and those who did not.

\section{Methods}

\section{Source of data}

More than 99\% of Taiwan's 23 million residents are covered by Taiwan's National Health Insurance Program, which has been in place since March 1995 (insurance claims data are available since 1996). Our research data were obtained from the reimbursement claims of Taiwan's National Health Insurance. The details of this database were described in our previous studies. ${ }^{7,23}$ The validity of the current database has been adequately evaluated, ${ }^{41-43}$ and research articles based on it have been published in prominent scientific journals worldwide. ${ }^{44-47}$

\section{Ethical approval}

Our research data were released for academic research access by Taiwan's National Health Insurance Research Database and Data Science Center, Ministry of Health and Welfare, and informed consent was not required because personal privacy was protected by using decoding and scrambling patients' identification. After critical evaluation, our study was approved by the Institutional Review Board of E-DA Hospital (EDA-JIRB-EMRP105144).

\section{Study design}

Among the 23 million residents of Taiwan, we identified $>200,000$ new stroke survivors aged $\geq 20$ years who were admitted to the hospital (people who received inpatient care with a physician's primary diagnosis of stroke) between January 1, 2000 and December 31, 2004. To confirm that all stroke patients in our study were incident cases, we included only new-onset stroke cases in the analysis, and those with a previous recorded stroke history within 4 years before the index date were excluded. In addition, new-onset stroke patients who had a history of epilepsy were excluded from this study. Among 96,131 eligible stroke survivors, 15,936 had received BYHWT at TCM clinics during the follow-up period after being admitted to hospital for stroke.

To avoid misclassification, we used a propensity score matching procedure (exposure vs non-exposure ratio $=1: 1$ ) to selected a non-BYHWT control group of stroke patients who did not receive TCM therapy. In the non-BYHWT control group, stroke patients were followed up from the discharge date of index stroke admission until December 31, 2009 or until the occurrence of an epileptic event, loss to follow-up, or death. To avoid immortal time bias (which might result in overestimation of the intervention's beneficial effects) in the BYHWT group, we followed up the stroke patients from the first date when they received BYHWT after index stroke admission until December 31, 2009 or until an epilepsy event, loss to follow-up, or death. The follow-up time, in person-years, was calculated for each stroke patient from the index date to the end point. We compared the risk of incident epilepsy during the follow-up period between those stroke patients who received BYHWT treatment and those who did not. After matching by propensity score, there were 8,971 pairs of stroke patients with and without BYHWT treatment.

\section{Criteria and definitions}

The formula BYHWT includes seven Chinese herbs: Huanqi (Radix Astragali seu Hedysari), Danggui (Radix Angelica sinensis), Chuanxiong (Rhizoma Ligustici Chuanxiong), Honghua (Flos Carthami), Dilong (Pheretima), Chishao (Radix Paeoniae Rubra), and Taoren (Semen Persicae). The BYHWT prescription in the clinical settings in Taiwan is concentrated Chinese herbal medicine which showed power type. The official recommendation for dosage is three times per day $(2.5 \mathrm{~g}$ per time), and the treatment duration is at least 6 days. TCM physicians consider every patient's symptoms and complaints and prescribe adequate treatment dosage and duration of BYHWT. According to the regulations of the National Health Insurance system, patients of low-income status were defined as patients who were qualified to waive co-payments for their medical expenditures. We calculated the population density (persons $/ \mathrm{km}^{2}$ ) of each administrative unit in Taiwan. Based on population density, these units were stratified into tertiles to designate areas of low, moderate, and high urbanization. We defined newly diagnosed stroke according to the International Classification of Diseases, Ninth Revision, Clinical Modification, as ICD-9-CM 430-437, a definition that has been validated in previous studies. ${ }^{15}$ The primary outcome, namely, the incidence of epilepsy, was defined as patients who sought medical care with a physician's primary diagnosis of epilepsy (ICD-9-CM 345). The ICD-9-CM was also used to 
identify coexisting medical conditions from medical claims for the follow-up period; these conditions included hypertension (ICD-9-CM 401-405), diabetes (ICD-9-CM 250), mental disorders (290-319), COPD (ICD-9-CM 491, 492, 496), ischemic heart disease (ICD-9-CM 410-414), urinary tract infection (ICD-9-CM 599.0), hyperlipidemia (ICD9-CM 272.0-272.4), pneumonia (ICD-9-CM 480-486), heart failure (ICD-9-CM 428), epilepsy (ICD-9-CM 345), and liver cirrhosis (ICD-9-CM 571). Renal dialysis was defined by the administration codes D8 and D9. Subtype of stroke (hemorrhage, occlusion and stenosis, transient ischemic attack, and others), use of antiepileptic drugs, whether the patient stayed in a medical center or not, length of hospital stay, admission to the intensive care unit, and receipt of neurosurgery were taken into consideration as baseline covariates between stroke patients with and without BYHWT.

\section{Statistical analysis}

We used a propensity score-matched pair design combined with frequency matching to balance the covariates (including age, sex, stroke subtype, low income, urbanization, admission to the intensive care unit, neurosurgery, length of hospital stay, subtypes of stroke, rehabilitation during follow-up, antiepileptic drugs, hypertension, diabetes, hyperlipidemia, mental disorders, cancer, head injuries, Parkinson's disease, renal dialysis, and Alzheimer's disease) between the BYHWT group and the nonBYHWT group. For achieving a balance of covariates within matched pairs, we performed a structured iterative approach to refine this logistic regression model using 1:1 case-control match on the propensity score. We then matched (without replacement) patients who had BYHWT with those who did not by using a greedy matching algorithm. The algorithm proceeds sequentially to the lowest digit match on propensity score (one digit). This will be referred to as the 8-1 digit match.

The frequency distributions of baseline characteristics were compared by chi-squared tests and Student's $t$-tests. We used multiple Cox proportional hazard regression models to calculate the adjusted HRs and 95\% CIs for epilepsy in stroke patients during the follow-up period according to BYHWT use. The association between BYHWT and post-stroke epilepsy in the subgroup analysis was also analyzed in the multiple Cox proportional hazard regression models. Additionally, the joint effects of acupuncture and BYHWT were estimated in further analysis by calculating adjusted HRs (95\% CIs).

\section{Results}

Due to the propensity score matching procedure, no significant differences in sex, age, low income, intensive care, neurosurgery, rehabilitation in follow-up, subtype of stroke, coexisting medical condition, antiepileptic drug, or length of hospital stay were noted between stroke patients who used BYHWT and those who did not (Table 1). Among the stroke patients who used BYHWT, 62.3\% were males and $56.1 \%$ were older people aged $\geq 65$ years. There were $70.8 \%$ stroke patients with ischemia (occlusion and stenosis) and most of them had hypertension (91.7\%).

During the follow-up period (Figure 1), the probability of epilepsy in patients receiving BYHWT was reduced compared to that in patients not receiving TCM. Among 17,942 stroke patients, 1,222 epilepsy events occurred during the follow-up period and 474 epilepsy events occurred in stroke patients receiving BYHWT (Table 2). The incidences of epilepsy in stroke patients with and without use of BYHWT were 11.6 and 16.1 per 1,000 person-years, respectively. After adjustment for all covariates listed in Table 1, stroke patients receiving BYHWT had a decreased risk of epilepsy (HR 0.69, 95\% CI 0.61-0.77) compared with those who did not receive BYHWT. The association between receiving BYHWT and reduced risk of post-stroke epilepsy was significant in women (HR 0.57, 95\% CI 0.47-0.70), men (HR 0.76, 95\% CI 0.66-0.87), and stroke patients aged 20-74 years, as well as in individuals who had experienced hemorrhagic stroke (HR 0.69, 95\% CI 0.50-0.96), occlusion or stenosis (HR 0.71, 95\% CI 0.62-0.82), transient ischemic attack (HR 0.41, 95\% CI 0.24-0.72), and other stroke types (HR 0.64, 95\% CI 0.46-0.90). The use of BYHWT was also associated with reduced post-stroke epilepsy in patients regardless of the length of their hospital stay (1-3, 4-6, $7-9$, or $\geq 10$ days), the number of medical conditions they had during follow-up $(0,1,2$, or $\geq 3)$, whether they stayed in an intensive care unit or not, and whether they attended rehabilitation or not. The subgroup showed that patients with transient ischemic attack had a very low incidence of epilepsy (5.5 per 1,000 person-years in BYHWT group and 12.4 per 1,000 person-years in the group without BYHWT) with an adjusted HR of 0.41 (95\% CI 0.24-0.72) associated with BYHWT use.

Among stroke patients, the risk of epilepsy showed a dose-dependent decrease as the quantity of BYHWT use increased (Table 3), from 1 package (HR 0.77, 95\% CI $0.66-0.90$ ) to $\geq 6$ packages (HR $0.52,95 \%$ CI $0.42-0.65$ ).

Compared to the non-BYHWT control group, stroke patients receiving BYHWT only (HR 0.69, 95\% CI, 0.580.83 ) or receiving BYHWT and acupuncture (HR 0.68, 95\% CI 0.60-0.78) exhibited a reduced incidence and risk of epilepsy (Table 4). 
Table I Comparison of the baseline characteristics between stroke patients with and without Bu Yang Huan Wu Tang

\begin{tabular}{|c|c|c|c|}
\hline & \multicolumn{2}{|c|}{ Bu Yang Huan Wu Tang } & \multirow{2}{*}{$\begin{array}{l}\text { Absolute } \\
\text { difference (\%) }\end{array}$} \\
\hline & No $(n=8,97 I)$ & Yes $(n=8,97 I)$ & \\
\hline Sex & $\mathrm{n}(\%)$ & $\mathrm{n}(\%)$ & 1.00 \\
\hline Female & $3,382(37.7)$ & $3,382(37.7)$ & \\
\hline Male & $5,589(62.3)$ & $5,589(62.3)$ & \\
\hline Age, years & & & 1.00 \\
\hline $20-44$ & $289(3.2)$ & $289(3.2)$ & \\
\hline $45-54$ & $1,232(13.7)$ & $\mathrm{I}, 232(13.7)$ & \\
\hline $55-64$ & $2,427(27.1)$ & $2,427(27.1)$ & \\
\hline $65-74$ & $3,43 I(38.3)$ & $3,431(38.3)$ & \\
\hline$\geq 75$ & $1,592(17.8)$ & $1,592(17.8)$ & \\
\hline Low income & $121(1.4)$ & $121(1.4)$ & 1.00 \\
\hline Intensive care unit stay & $7 \mid 4(8.0)$ & $714(8.0)$ & 1.00 \\
\hline Neurosurgery & $119(1.3)$ & $119(1.3)$ & 1.00 \\
\hline Length of stay, days & & & 1.00 \\
\hline $\mathrm{I}-3$ & $1,685(18.8)$ & $\mathrm{I}, 685(\mathrm{I} 8.8)$ & \\
\hline $4-6$ & $2,875(32.1)$ & $2,875(32.1)$ & \\
\hline $7-9$ & $1,880(21.0)$ & $\mathrm{I}, 880(2 \mathrm{I} .0)$ & \\
\hline$\geq 10$ & 2,53 I (28.2) & $2,531(28.2)$ & \\
\hline Mean \pm SD & $8.06 \pm 5.89$ & $8.07 \pm 5.93$ & 0.12 \\
\hline Subtypes of stroke & & & 1.00 \\
\hline Hemorrhage & $780(8.7)$ & $780(8.7)$ & \\
\hline Occlusion and stenosis & $6,348(70.8)$ & $6,348(70.8)$ & \\
\hline Transient ischemic attack & $743(8.3)$ & $743(8.3)$ & \\
\hline Others & $1,100(12.3)$ & $1,100(12.3)$ & \\
\hline Intensive rehabilitation & $4,886(54.5)$ & $4,886(54.5)$ & 1.00 \\
\hline Basic rehabilitation & $4,344(48.4)$ & $4,344(48.4)$ & \\
\hline Antiepileptic drug & $2,589(28.9)$ & $2,589(28.9)$ & 1.00 \\
\hline \multicolumn{4}{|l|}{ Coexisting medical condition } \\
\hline Hypertension & $8,226(91.7)$ & $8,226(91.7)$ & 1.00 \\
\hline Diabetes & $3,858(43.0)$ & $3,858(43.0)$ & 1.00 \\
\hline Hyperlipidemia & $3,734(41.6)$ & $3,734(41.6)$ & 1.00 \\
\hline Mental disorder & 2,978 (33.2) & $2,978(33.2)$ & 1.00 \\
\hline Cancer & $643(7.2)$ & $643(7.2)$ & 1.00 \\
\hline Head injury & $557(6.2)$ & $557(6.2)$ & 1.00 \\
\hline Parkinson's disease & $235(2.6)$ & $235(2.6)$ & 1.00 \\
\hline Renal dialysis & $108(1.2)$ & $108(1.2)$ & 1.00 \\
\hline Alzheimer's disease & $6(0.1)$ & $6(0.1)$ & 1.00 \\
\hline
\end{tabular}

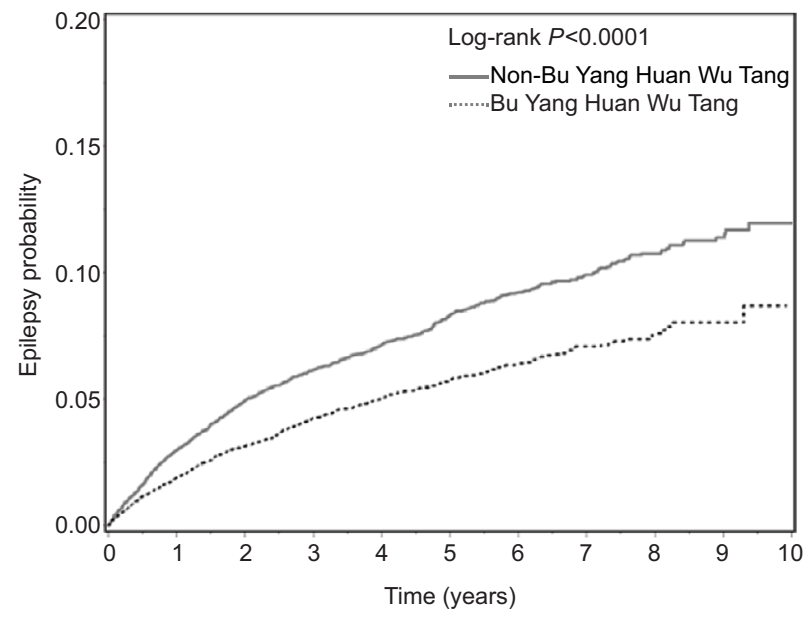

Figure I The estimated epilepsy probability of stroke patients with and without Bu Yang Huan Wu Tang using the Kaplan-Meier method (log-rank test, $P<0.000 \mathrm{I}$ ).
The association between days of BYHWT treatment and reduced risk of post-stroke epilepsy was significant when the decoction was taken for $>6$ days (Table S1). The top formula and herbal medicine prescribed to accompany BYHWT were Tianma Gouteng Decoction and Dan Shen, respectively (Table S2). The top ten formulas and the top ten herbs prescribed to stroke patients in combination with BYHWT during the follow-up period are shown in Table S3.

\section{Discussion}

To our knowledge, the present study is the first to use nationwide data, propensity score matching, and a retrospective cohort study design to report a significant association between use of BYHWT and reduced risk of post-stroke epilepsy, and this beneficial effect could be observed in 
Table 2 Epilepsy risk and subgroup analyses between stroke patients with and without BYHWT

\begin{tabular}{|c|c|c|c|c|c|c|c|}
\hline & \multicolumn{3}{|c|}{ No BYHWT $(n=8,97 I)$} & \multicolumn{3}{|c|}{ BYHWT $(n=8,97 I)$} & \multirow[t]{2}{*}{ HR $(95 \% \mathrm{Cl})^{\mathrm{b}}$} \\
\hline & Events & PYs & $\mathbf{I R}^{\mathbf{a}}$ & Events & PYs & $\mathbf{I R}^{\mathbf{a}}$ & \\
\hline All & 748 & 46,455 & 16.1 & 474 & 41,013 & 11.6 & $0.69(0.6 \mathrm{I}-0.77)$ \\
\hline Female & 286 & 17,632 & 16.2 & 149 & 15,503 & 9.6 & $0.57(0.47-0.70)$ \\
\hline Male & 462 & 28,823 & 16.0 & 325 & 25,510 & 12.7 & $0.76(0.66-0.87)$ \\
\hline \multicolumn{8}{|l|}{ Age, years } \\
\hline $20-44$ & 31 & 1,618 & 19.2 & 20 & $\mathrm{I}, 364$ & 14.7 & $0.68(0.38-1.18)$ \\
\hline $45-54$ & 96 & 7,277 & 13.2 & 62 & 5,933 & 10.5 & $0.72(0.52-0.99)$ \\
\hline $55-64$ & 203 & $|3,57|$ & 15.0 & 104 & $\mathrm{II}, 503$ & 9.0 & $0.57(0.45-0.73)$ \\
\hline $65-74$ & 292 & 17,332 & 16.8 & 194 & 15,943 & 12.2 & $0.70(0.58-0.84)$ \\
\hline$\geq 75$ & 126 & 6,657 & 18.9 & 94 & 6,271 & 15.0 & $0.78(0.60-1.02)$ \\
\hline No rehabilitation & 252 & 16,539 & 15.2 & 150 & $|4,33|$ & 10.5 & $0.64(0.52-0.78)$ \\
\hline Rehabilitation & 496 & 29,917 & 16.6 & 324 & 26,681 & 12.1 & $0.7 \mathrm{I}(0.62-0.8 \mathrm{I})$ \\
\hline \multicolumn{8}{|l|}{ Medical conditions } \\
\hline 0 & 66 & 778 & 84.8 & 36 & 1,088 & 33.1 & $0.43(0.29-0.64)$ \\
\hline 1 & 221 & 8,621 & 25.6 & 158 & 8,462 & 18.7 & $0.70(0.57-0.86)$ \\
\hline 2 & 262 & 15,907 & 16.5 & 146 & $|4,24|$ & 10.3 & $0.60(0.49-0.73)$ \\
\hline$\geq 3$ & 199 & 21,149 & 9.4 & 134 & 17,222 & 7.8 & $0.83(0.66-1.03)$ \\
\hline \multicolumn{8}{|l|}{ Length of stay, days } \\
\hline $\mathrm{I}-3$ & 121 & 9,113 & 13.3 & 75 & 7,571 & 9.9 & $0.69(0.52-0.92)$ \\
\hline $4-6$ & 236 & 15,418 & 15.3 & 137 & 13,079 & 10.5 & $0.66(0.53-0.8 I)$ \\
\hline $7-9$ & 125 & 9,994 & 12.5 & 79 & 8,758 & 9.0 & $0.68(0.5 \mathrm{I}-0.90)$ \\
\hline$\geq 10$ & 266 & $|1,93|$ & 22.3 & 183 & 11,604 & 15.8 & $0.69(0.58-0.84)$ \\
\hline \multicolumn{8}{|l|}{ Subtypes of stroke } \\
\hline Hemorrhage & 92 & 4,067 & 22.6 & 61 & 3,726 & 16.4 & $0.69(0.50-0.96)$ \\
\hline Occlusion/stenosis & 512 & 32,523 & 15.7 & 342 & 29,262 & 11.7 & $0.71(0.62-0.82)$ \\
\hline TIA & 52 & 4,179 & 12.4 & 17 & 3,113 & 5.5 & $0.4 I(0.24-0.72)$ \\
\hline Others & 92 & 5,687 & 16.2 & 54 & 4,913 & 11.0 & $0.64(0.46-0.90)$ \\
\hline ICU stay & 100 & 3,326 & 30.1 & 64 & 3,319 & 19.3 & $0.63(0.46-0.86)$ \\
\hline No ICU stay & 648 & 43,129 & 15.0 & 410 & 34,694 & 10.9 & $0.69(0.6 \mathrm{I}-0.78)$ \\
\hline
\end{tabular}

Notes: aPer I,000 person-years. ' ${ }^{\circ}$ Calculated by univariate Cox proportional hazard model.

Abbreviations: BYHWT, Bu Yang Huan Wu Tang; ICU, intensive care unit; IR, incidence rate; PY, person-year; TIA, transient ischemic attack.

Table 3 Risk of epilepsy in stroke patients during the follow-up period by the number of packages of Bu Yang Huan Wu Tang

\begin{tabular}{|l|l|l|l|l|l|}
\hline $\begin{array}{l}\text { Number of } \\
\text { packages }\end{array}$ & $\mathbf{n}$ & Events & Person-year & Incidence & HR (95\% CI) \\
\hline 0 & 8,971 & 748 & 46,455 & 16.1 & \\
\hline 1 & 3,603 & 202 & 15,321 & 13.2 & $1.00($ reference $)$ \\
\hline 2 & 1,538 & 93 & 6,869 & 13.5 & $0.77(0.66-0.90)$ \\
\hline 3 & 856 & 48 & 4,110 & 11.7 & $0.80(0.64-0.99)$ \\
\hline 4 & 544 & 28 & 2,601 & 10.8 & $0.70(0.52-0.94)$ \\
\hline 5 & 417 & 16 & 1,978 & 8.1 & $0.65(0.44-0.94)$ \\
\hline$\geq 6$ & 2,013 & 87 & 10,134 & 8.6 & $0.48(0.29-0.79)$ \\
\hline
\end{tabular}

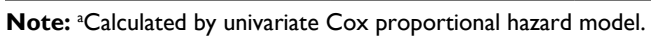

various subgroups of stroke patients. Further analysis also revealed a graded biological relationship between the number of packages of BYHWT used and the risk of poststroke epilepsy.

Age, sex, low income, hypertension, hyperlipidemia, diabetes mellitus, and mental disorder were the factors associated with stroke. ${ }^{24}$ These sociodemographic factors and medical conditions are also associated with epilepsy. ${ }^{25}$
In addition, neurosurgery, intensive care, and length of hospital stay also impact the incidence of post-stroke epilepsy. ${ }^{26}$ To reduce the confounding effects of these strokerelated and epilepsy-related factors, we used a matching procedure with propensity scores to control these potential confounders.

Our stratification analysis revealed that the association between BYHWT treatment and reduced risk 
Table 4 The joint effects of BYHWT and acupuncture treatment on the risk of epilepsy in stroke patients during the follow-up period

\begin{tabular}{|l|l|l|l|l|}
\hline $\begin{array}{l}\text { Treatment } \\
\text { combination }\end{array}$ & $\mathbf{n}$ & Cases & Incidence $^{\mathbf{a}}$ & HR $(\mathbf{9 5} \% \mathbf{C l})^{\mathbf{b}}$ \\
\hline No BYHWT & $8,97 \mathrm{I}$ & 748 & 16.1 & 1.00 (reference) \\
\hline BYHWT only & 2,938 & 149 & $\mathrm{II} .9$ & $0.69(0.58-0.83)$ \\
\hline $\begin{array}{l}\text { BYHWT and } \\
\text { acupuncture }\end{array}$ & 6,033 & 325 & 11.4 & $0.68(0.60-0.78)$ \\
\hline
\end{tabular}

Notes: aPer I,000 person-years. ${ }^{\circ}$ Calculated by univariate Cox proportional hazard model.

Abbreviation: BYHWT, Bu Yang Huan Wu Tang.

of post-stroke epilepsy remained significant in 20- to 74-year-old stroke patients, both sexes, and every stroke subtype, regardless of the number of other medical conditions present. In addition, stroke patients who received BYHWT either alone or in combination with acupuncture had a significantly decreased risk of epilepsy. Despite the suboptimal sensitivity of this study, our findings still provide grounds for a possible relationship between BYHWT treatment and reduced risk of post-stroke epilepsy from the viewpoint of epidemiology.

In a rat model of ischemia-reperfusion injury, BYHWT treatment improved the nerve function, and the beneficial effects greatly increased as the dose of BYHWT increased. ${ }^{27}$ A similar dose-response relationship was also observed in our study: the incidence of post-stroke epilepsy decreased as the dose of BYHWT increased, and patients who used the treatment for $>36$ days had a lower risk of post-stroke epilepsy than those who used it for 5 or fewer days. However, well-designed prospective studies or clinical trials will be necessary to provide solid evidence for the graded, beneficial biological effects of BYHWT.

BYHWT is used to treat a variety of diseases, including stroke, vertigo, vascular dementia, coronary heart disease, and chemotherapy-induced neuropathy ${ }^{28,29} \mathrm{~A}$ systematic review suggested that BYHWT is generally safe and can improve neurological deficits for acute ischemic stroke patients. ${ }^{22}$ However, previous results have been insufficient to support the routine use of BYHWT for acute stroke due to the poor methodological quality of the studies..$^{22}$ Departing from the existing literature, we conducted a nationwide study with a large sample size, propensity score matching, immortal time adjustment, and multivariate Cox proportional hazard models. Our study, with its improved methodological quality, raised the possibility that BYHWT may be beneficial in reducing the risk of epilepsy in stroke patients.

Although post-stroke hypoxia, metabolic dysfunction, inflammatory responses, and blood-brain barrier damage were identified as factors associated with post-stroke epilepsy, ${ }^{5,30}$ epileptogenesis itself is still quite a poorly known phenomenon. A previous study suggested that BYHWT may restore the brain function and improve neurological deficits in a mouse model of ischemic stroke. ${ }^{31}$ In the same study, BYHWT also reduced inflammation, oxidative stress, and apoptosis, in addition to improving neurogenesis. ${ }^{31}$

In general, BYHWT includes seven Chinese herbs, and the effects of its active components have been widely reported as follows. The major herb of BYHWT is Huanqi, which is beneficial for patients with post-stroke fatigue and improves the functional outcomes of hemorrhagic stroke patients. ${ }^{32,33}$ The major active component of Huanqi is astragaloside IV that has neuroprotective properties stemming from antioxidative, antiapoptotic, and antiinflammatory actions. ${ }^{34,35}$ Tetramethylpyrazine, found in Chuanxiong, has been shown to have anti-inflammatory and neuroprotective effects in an experimental study. ${ }^{36}$ Ferulic acid, found in Danggui and Chuanxiong, has antioxidative properties that provide neuroprotective effects. ${ }^{37}$ Hydroxysafflor yellow A, found in Honghua, provides neuroprotection against cerebral injury by modulating inflammatory and apoptotic processes. ${ }^{38}$ In addition, Dilong, Chishao, and Taoren have beneficial effects on cerebral ischemic injury. ${ }^{39}$ The above experimental evidence provides possible explanations for the beneficial neuroprotective effects of BYHWT in stroke patients. Possible therapeutic effects of the components of BYHWT are listed in Table S4.

However, there is still lack of reports on the molecules and pathways involved in the mechanisms of BYHWT. It may not be safe and scientifically sound to promote some therapies without clear safety data and mechanisms. We seriously suggest that people using BYHWT should fully comply with professional physician's directions. Further repeatable, randomized, double-blinded experiments will be necessary to establish solid evidence of the benefits of BYHWT and characterize its mechanisms in humans.

\section{Limitations}

First, we used insurance claims data, which lack information on the clinical risk scores (such as the National Institute of Health Stroke Scale or the Barthel index), lesion characteristics (location and size), biochemical measures (such as glucose, total cholesterol, and high-sensitivity C-reactive protein), and patients' lifestyle (such as smoking, alcohol drinking, and physical activity). Cortical stroke location is also an important risk factor for post-stroke epilepsy, but our study was limited by the unavailable information on 
this. ${ }^{48}$ Second, although the diagnosis codes of diseases and medications are validated to be accurate, ${ }^{40}$ patients with mild symptoms of epilepsy may simply not seek medical care. Third, out-of-pocket purchases of Chinese herbal remedies and over-the-counter herbal medicines could not be evaluated because we used only the claims data of the National Health Insurance. Fourth, physician's prescription is not equal to patient's intake. The use of BYHWT in this study is physician's prescription, which may not reflect the actual use of the medication in stroke patients. Fifth, those who take the medications as directed may also be more likely to do more to improve their health and recovery. The beneficial effects in reducing epilepsy among stroke patients in this study are not all derived from BYHWT. Fifth, we could not exclude the possibility of selection bias because the control group (stroke patients without using BYHWT) was selected by the baseline characteristics of the treatment group (stroke patients using BYHWT) with matching procedure by propensity score. In addition, the doses of BYHWT and other combined Chinese herbal remedies for patients with stroke varied across TCM physicians. We could not exclude the potential therapeutic effects of other prescribed Chinese herbal medicines in stroke patients. The possible side effects or adverse effects of the components of BYHWT should also not be ignored in this study (Table S5). Finally, residual confounding may exist in our study, despite the comprehensive matching and multiple regression adjustment. The severity of stroke (stroke-related comorbidities, clinical risk scores, lesion characteristics, and cortical stroke location) is an important confounding factor that was not considered in our study, and several potential confounders (detailed medication use, biochemical measures, lifestyles, socioeconomic status, genetics, family history, and use of other folk therapies) were not matched or adjusted in our analysis. All the positive effects of BYHWT in stroke patients in this study might not be due to BYHWT itself. Better lifestyle habits and using other conventional treatment (Western medicine) also contribute to reducing post-stroke epilepsy.

\section{Conclusion}

Our study suggests that stroke patients receiving BYHWT treatment exhibited a reduced risk of post-stroke epilepsy compared to patients who did not receive BYHWT, and this association could be observed in various subgroups. However, well-designed, high-quality randomized controlled trials are needed to confirm the beneficial effect of BYHWT on the risk of post-stroke epilepsy.

\section{Acknowledgment}

This study was supported in part by Taiwan's Ministry of Science and Technology (MOST106-2314-B-038-036-MY3, MOST106-2320-B-214-003).

\section{Author contributions}

All authors contributed to data analysis, drafting or revising the article, gave final approval of the version to be published, and agree to be accountable for all aspects of the work.

\section{Disclosure}

The authors report no conflicts of interest in this work.

\section{References}

1. GBD 2016 DALYs and HALE Collaborators. Global, regional, and national disability-adjusted life-years (DALYs) for 333 diseases and injuries and healthy life expectancy (HALE) for 195 countries and territories, 1990-2016: a systematic analysis for the Global Burden of Disease Study 2016. Lancet. 2017;390(10100):1260-1344.

2. Luengo-Fernandez R, Gray AM, Rothwell PM. Costs of stroke using patient-level data: a critical review of the literature. Stroke. 2009;40(2):e18-e23.

3. Balami JS, Chen RL, Grunwald IQ, Buchan AM. Neurological complications of acute ischaemic stroke. Lancet Neurol. 2011;10(4):357-371.

4. Ingeman A, Andersen G, Hundborg HH, Svendsen ML, Johnsen SP. In-hospital medical complications, length of stay, and mortality among stroke unit patients. Stroke. 2011;42(11):3214-3218.

5. Pitkänen A, Roivainen R, Lukasiuk K. Development of epilepsy after ischaemic stroke. Lancet Neurol. 2016;15(2):185-197.

6. Qian X, Zhou X, You Y, et al. Traditional Chinese acupuncture for poststroke depression: a single-blind double-simulated randomized controlled trial. J Altern Complement Med. 2015;21(12):748-753.

7. Shih CC, Liao CC, Sun MF, et al. A retrospective cohort study comparing stroke recurrence rate in ischemic stroke patients with and without acupuncture treatment. Medicine. 2015;94(39):e1572.

8. Beghi E, D'Alessandro R, Beretta S, et al. Incidence and predictors of acute symptomatic seizures after stroke. Neurology. 2011;77(20): $1785-1793$.

9. Graham NS, Crichton S, Koutroumanidis M, Wolfe CD, Rudd AG. Incidence and associations of poststroke epilepsy: the prospective South London Stroke Register. Stroke. 2013;44(3):605-611.

10. Arntz RM, Rutten-Jacobs LC, Maaijwee NA, et al. Poststroke epilepsy is associated with a high mortality after a stroke at young age: follow-up of transient ischemic attack and stroke patients and unelucidated risk factor evaluation study. Stroke. 2015;46(8):2309-2311.

11. Shih CC, Su YC, Liao CC, Lin JG. Patterns of medical pluralism among adults: results from the 2001 National Health Interview Survey in Taiwan. BMC Health Serv Res. 2010;10:191.

12. Yeh YH, Chou YJ, Huang N, Pu C, Chou P. The trends of utilization in traditional Chinese medicine in Taiwan from 2000 to 2010: A population-based study. Medicine. 2016;95(27):e4115.

13. Park SU, Cho SY, Park JM, et al. Integrative treatment modalities for stroke victims in Korea. Complement Ther Clin Pract. 2014;20(1):37-41.

14. Liao CC, Lin JG, Tsai CC, et al. An investigation of the use of traditional Chinese medicine in stroke patients in Taiwan. Evid Based Complement Alternat Med. 2012;2012:387164.

15. Chang CC, Chen TL, Chiu HE, et al. Outcomes after stroke in patients receiving adjuvant therapy with traditional Chinese medicine: a nationwide matched interventional cohort study. $J$ Ethnopharmacol. 2016;177:46-52. 
16. Chang CC, Lee YC, Lin CC, et al. Characteristics of traditional Chinese medicine usage in patients with stroke in Taiwan: a nationwide population-based study. J Ethnopharmacol. 2016;186:311-321.

17. Hung IL, Hung YC, Wang LY, et al. Chinese herbal products for ischemic stroke. Am J Chin Med. 2015;43(7):1365-1379.

18. Chen HJ, Shen YC, Shiao YJ, et al. Multiplex brain proteomic analysis revealed the molecular therapeutic effects of Buyang Huanwu decoction on cerebral ischemic stroke mice. PLoS One. 2015;10(10): e0140823.

19. Luo L, Deng S, Yi J, et al. Buyang Huanwu decoction ameliorates poststroke depression via promoting neurotrophic pathway mediated neuroprotection and neurogenesis. Evid Based Complement Alternat Med. 2017;2017:4072658.

20. Pan R, Cai J, Zhan L, et al. Buyang Huanwu decoction facilitates neurorehabilitation through an improvement of synaptic plasticity in cerebral ischemic rats. BMC Complement Altern Med. 2017;17(1): 173.

21. Wei RL, Teng HJ, Yin B, et al. A systematic review and meta-analysis of buyang huanwu decoction in animal model of focal cerebral ischemia. Evid Based Complement Alternat Med. 2013;2013:138484.

22. Hao CZ, Wu F, Shen J, et al. Clinical efficacy and safety of buyang huanwu decoction for acute ischemic stroke: a systematic review and meta-analysis of 19 randomized controlled trials. Evid Based Complement Alternat Med. 2012;2012:630124.

23. Shih CC, Yeh CC, Hu CJ, et al. Risk of dementia in patients with nonhaemorrhagic stroke receiving acupuncture treatment: a nationwide matched cohort study from Taiwan's National Health Insurance Research Database. BMJ Open. 2017;7(6):e013638.

24. Shigematsu K, Watanabe Y, Nakano H; Kyoto Stroke Registry Committee. Influences of hyperlipidemia history on stroke outcome; a retrospective cohort study based on the Kyoto Stroke Registry. BMC Neurol. 2015; $15: 44$.

25. Mehta A, Zusman BE, Choxi R, et al. Seizures after intracerebral hemorrhage: incidence, risk factors, and impact on mortality and morbidity. World Neurosurg. 2018;112:e385-e392.

26. Huang CW, Saposnik G, Fang J, Steven DA, Burneo JG. Influence of seizures on stroke outcomes: a large multicenter study. Neurology. 2014;82(9):768-776.

27. Wang L, Huang Y, Wu J, et al. Effect of Buyang Huanwu decoction on amino acid content in cerebrospinal fluid of rats during ischemic/ reperfusion injury. J Pharm Biomed Anal. 2013;86:143-150.

28. Schröder S, Beckmann K, Franconi G, et al. Can medical herbs stimulate regeneration or neuroprotection and treat neuropathic pain in chemotherapy-induced peripheral neuropathy? Evid Based Complement Alternat Med. 2013;2013:423713.

29. Li JH, Liu AJ, Li HQ, Jh L, Hq L. Buyang Huanwu decoction for healthcare: evidence-based theoretical interpretations of treating different diseases with the same method and target of vascularity. Evid Based Complement Alternat Med. 2014;2014:506783.

30. Tanaka T, Ihara M. Post-stroke epilepsy. Neurochem Int. 2017;107: 219-228.

31. Wang HW, Liou KT, Wang YH, et al. Deciphering the neuroprotective mechanisms of Bu-yang Huan-wu decoction by an integrative neurofunctional and genomic approach in ischemic stroke mice. $J$ Ethnopharmacol. 2011;138(1):22-33.
32. Liu CH, Tsai CH, Li TC, et al. Effects of the traditional Chinese herb Astragalus membranaceus in patients with poststroke fatigue: a doubleblind, randomized, controlled preliminary study. J Ethnopharmacol. 2016;194:954-962.

33. Chen CC, Lee HC, Chang JH, et al. Chinese herb Astragalus membranaceus enhances recovery of hemorrhagic stroke: double-blind, placebo-controlled, randomized study. Evid Based Complement Alternat Med. 2012;2012:708452.

34. Shao A, Guo S, Tu S, et al. Astragaloside IV alleviates early brain injury following experimental subarachnoid hemorrhage in rats. Int J Med Sci. 2014;11(10):1073-1081.

35. Wang HL, Zhou QH, Xu MB, Zhou XL, Zheng GQ, Mb X. Astragaloside IV for experimental focal cerebral ischemia: preclinical evidence and possible mechanisms. Oxid Med Cell Longev. 2017;2017:8424326.

36. Kao TK, Chang CY, Ou YC, et al. Tetramethylpyrazine reduces cellular inflammatory response following permanent focal cerebral ischemia in rats. Exp Neurol. 2013;247:188-201.

37. Koh PO. Ferulic acid attenuates the injury-induced decrease of protein phosphatase 2A subunit B in ischemic brain injury. PLoS One. 2013;8(1):e54217.

38. Deng L, Wan H, Zhou H, Yu L, He Y. Protective effect of hydroxysafflor yellow A alone or in combination with acetylglutamine on cerebral ischemia reperfusion injury in rat: a PET study using ${ }^{18} \mathrm{~F}$-fuorodeoxyglucose. Eur J Pharmacol. 2018;825:119-132.

39. Mu Q, Liu P, Hu X, Gao H, Zheng X, Huang H. Neuroprotective effects of Buyang Huanwu decoction on cerebral ischemia-induced neuronal damage. World Neurosurg. 2018;112:e385-e392.

40. Cheng CL, Kao YH, Lin SJ, Lee CH, Lai ML. Validation of the National Health Insurance Research Database with ischemic stroke cases in Taiwan. Pharmacoepidemiol Drug Saf. 2011;20(3):236-242.

41. Cheng CL, Chien HC, Lee CH, Lin SJ, Yang YH. Validity of in-hospital mortality data among patients with acute myocardial infarction or stroke in National Health Insurance Research Database in Taiwan. Int J Cardiol. 2015;201:96-101.

42. Cheng CL, Kao YH, Lin SJ, Lee CH, Lai ML. Validation of the National Health Insurance Research Database with ischemic stroke cases in Taiwan. Pharmacoepidemiol Drug Saf. 2011;20(3):236-242.

43. Hsieh CY, Chen CH, Li CY, Lai ML. Validating the diagnosis of acute ischemic stroke in a National Health Insurance claims database. $J$ Formos Med Assoc. 2015;114(3):254-259.

44. Yeh CC, Wang HH, Chou YC, et al. High risk of gastrointestinal hemorrhage in patients with epilepsy: a nationwide cohort study. Mayo Clin Proc. 2013;88(10):1091-1098.

45. Yeh CC, Chen TL, Hu CJ, Chiu WT, Liao CC. Risk of epilepsy after traumatic brain injury: a retrospective population-based cohort study. J Neurol Neurosurg Psychiatry. 2013;84(4):441-445.

46. Liao CC, Chang PY, Yeh CC, et al. Outcomes after surgery in patients with previous stroke. Br J Surg. 2014;101(12):1616-1622.

47. Liao CC, Chou YC, Yeh CC, Hu CJ, Chiu WT, Chen TL. Stroke risk and outcomes in patients with traumatic brain injury: 2 nationwide studies. Mayo Clin Proc. 2014;89(2):163-172.

48. Okuda S, Takano S, Ueno M, Hamaguchi H, Kanda F. Clinical features of lateonset poststroke seizures. J Stroke Cerebrovasc Dis. 2012;21(7):583-586.

49. Chu S, Mao XD, Wang L, Peng W. Effects of Huang Qi decoction on endothelial dysfunction induced by homocysteine. Evid Based Complement Alternat Med. 2016;2016:7272694. 


\section{Supplementary materials}

Table SI Comparison of baseline characteristics between stroke patients with and without Bu Yang Huan Wu Tang before matching procedure

\begin{tabular}{|c|c|c|c|}
\hline & \multicolumn{2}{|c|}{ Bu Yang Huan Wu Tang } & \multirow{2}{*}{$\begin{array}{l}\text { Absolute } \\
\text { difference (\%) }\end{array}$} \\
\hline & No $(n=80,195)$ & Yes $(n=15,936)$ & \\
\hline Sex & n (\%) & n (\%) & \\
\hline Female & $33,429(41.7)$ & $6,373(40.0)$ & 4.08 \\
\hline Male & $46,766(58.3)$ & $9,563(60.0)$ & 2.83 \\
\hline \multicolumn{4}{|l|}{ Age, years } \\
\hline $20-44$ & $2,407(3.0)$ & $\mathrm{I}, 057(6.6)$ & 54.55 \\
\hline $45-54$ & $6,650(8.3)$ & $3,021(19.0)$ & 56.32 \\
\hline $55-64$ & $|2,54|(\mid 5.6)$ & $4,463(28.0)$ & 44.29 \\
\hline $65-74$ & $25,913(32.3)$ & $5,111(32.1)$ & 0.31 \\
\hline$\geq 75$ & $32,684(40.8)$ & $2,284(14.3)$ & 64.95 \\
\hline Low income & $4,339(5.4)$ & $823(5.2)$ & 3.70 \\
\hline Intensive care unit stay & II,84I (I4.8) & $2,423(15.2)$ & 2.63 \\
\hline Neurosurgery & $2,505(3.1)$ & $506(3.2)$ & 3.13 \\
\hline Length of stay, days & & & 1.00 \\
\hline $\mathrm{I}-3$ & $\mathrm{I}, 685(\mathrm{I} 8.8)$ & $\mathrm{I}, 685(18.8)$ & \\
\hline $4-6$ & $2,875(32.1)$ & $2,875(32.1)$ & \\
\hline $7-9$ & $\mathrm{I}, 880(21.0)$ & $\mathrm{I}, 880(21.0)$ & \\
\hline$\geq 10$ & $2,53 \mid(28.2)$ & $2,53 \mid(28.2)$ & \\
\hline Mean \pm SD & $8.91 \pm 6.90$ & $8.97 \pm 6.75$ & 0.67 \\
\hline \multicolumn{4}{|l|}{ Subtypes of stroke } \\
\hline Hemorrhage & $8,800(11.0)$ & $2,564(16.1)$ & 31.68 \\
\hline Occlusion and stenosis & $47,247(58.9)$ & $9,712(60.9)$ & 3.28 \\
\hline Transient ischemic attack & $10,940(13.6)$ & $\mathrm{I}, 43 \mathrm{I}(9.0)$ & 33.82 \\
\hline Others & $13,208(16.5)$ & $2,229(14.0)$ & 15.15 \\
\hline Intensive rehabilitation & $29,618(36.9)$ & $9,546(59.9)$ & 38.40 \\
\hline Basic rehabilitation & $27,597(34.4)$ & $8,67 \mid(54.4)$ & 36.76 \\
\hline Antiepileptic drug & $21,975(27.4)$ & $6,010(37.7)$ & 27.32 \\
\hline \multicolumn{4}{|l|}{ Coexisting medical condition } \\
\hline Hypertension & $60,364(75.3)$ & $14,094(88.4)$ & 14.82 \\
\hline Diabetes & $30,935(38.6)$ & $6,913(43.4)$ & 11.09 \\
\hline Hyperlipidemia & $20,272(25.3)$ & $7,488(47.0)$ & 46.17 \\
\hline Mental disorder & $24,317(30.3)$ & $6,591(41.4)$ & $26.8 I$ \\
\hline Cancer & $9,388(11.7)$ & $\mathrm{I}, 8 \mathrm{I} 5(\mathrm{II} .4)$ & 2.56 \\
\hline Head injury & $8,40 \mathrm{I}(\mathrm{I} 0.5)$ & $2,175(I 3.7)$ & 23.36 \\
\hline Parkinson's disease & $4,20 \mathrm{I}(5.2)$ & $995(6.2)$ & 16.13 \\
\hline Renal dialysis & $3,313(4.1)$ & $413(2.6)$ & 36.59 \\
\hline Alzheimer's disease & $512(0.6)$ & $69(0.4)$ & 33.33 \\
\hline
\end{tabular}

Table S2 Risk of post-stroke epilepsy during the follow-up period treatment days of BYHWT

\begin{tabular}{|c|c|c|c|c|c|}
\hline BYHWT & $\mathbf{n}$ & Events & Person-years & Incidence & HR $(95 \% \mathrm{Cl}) *$ \\
\hline No BYHWT & 8,971 & 748 & 46,455 & 16.1 & 1.00 (reference) \\
\hline \multicolumn{6}{|c|}{ Cumulative treatment, days } \\
\hline $\mathrm{I}-7$ & 3,382 & 189 & 14,402 & 13.1 & $0.77(0.65-0.90)$ \\
\hline $8-14$ & 1,520 & 92 & $6,87 I$ & 13.4 & $0.79(0.64-0.98)$ \\
\hline$|5-2|$ & 870 & 45 & 4,143 & 10.9 & $0.65(0.48-0.88)$ \\
\hline $22-25$ & 536 & 28 & 2,524 & $1 \mathrm{II} . \mathrm{I}$ & $0.66(0.45-0.97)$ \\
\hline $29-35$ & 429 & 21 & 2,019 & 10.5 & $0.62(0.40-0.96)$ \\
\hline $36-42$ & 340 & 18 & $\mathrm{I}, 557$ & 11.6 & $0.68(0.43-1.09)$ \\
\hline $43-49$ & 222 & 6 & $\mathrm{I}, 068$ & 5.6 & $0.34(0.15-0.75)$ \\
\hline$\geq 50$ & 1,672 & 75 & 8,428 & 8.9 & $0.54(0.42-0.68)$ \\
\hline
\end{tabular}

Note: *Adjusted for all covariates listed in Table I.

Abbreviation: BYHWT, Bu Yang Huan Wu Tang. 
Table S3 The top ten formulas and the top ten herbs prescribed to stroke patients in combination with Bu Yang Huan Wu Tang during the follow-up period

\begin{tabular}{|c|c|c|c|c|c|}
\hline Herbs & Frequency & $\%$ & Formulas & Frequency & $\%$ \\
\hline Dan Shen & 56,629 & 4.97 & Tian Ma Gou Teng Yin & 27,907 & 3.67 \\
\hline San Qi & 26,521 & 2.33 & Ma Zi Ren Wan & 21,751 & 2.86 \\
\hline Du Zhong & 22,997 & 2.02 & Xue Fu Zhu Yu Tang & 21,728 & 2.86 \\
\hline Da Huang & 21,248 & 1.86 & Shu Jing Huo Xie Tang & 19,747 & 2.60 \\
\hline Niu Xi & 21,033 & 1.85 & Du Huo Ji Sheng Tang (Wan) & 16,070 & 2.11 \\
\hline Tian Ma & 19,477 & 1.71 & Ji Sheng Shen Qi Wan & 15,215 & 2.00 \\
\hline Huang Qi & 18,148 & 1.59 & Sheng Mai San & 14,980 & 1.97 \\
\hline Ge Gen & 18,106 & 1.59 & Liu Wei Di Huang San (Wan) & $|4,50|$ & 1.91 \\
\hline Xu Duan & 14,437 & 1.27 & Zhi Gan Cao Tang & 13,542 & 1.78 \\
\hline ji Xue Teng & 14,310 & 1.26 & Qi Ju Di Huang Wan & $\mathrm{II}, 794$ & 1.55 \\
\hline
\end{tabular}

Table S4 Possible therapeutic effects of components of Bu Yang Huan Wu Tang

\begin{tabular}{|c|c|c|}
\hline English name & Official name & Possible therapeutic effects \\
\hline Huanqi ${ }^{1,2}$ & Astragali Radix & $\begin{array}{l}\text { Enhances immunity, inhibits the formation of lipid peroxides in the myocardium, decreases blood } \\
\text { coagulation, has increased efficacy against vesicular stomatitis }\end{array}$ \\
\hline Danggui ${ }^{3,4}$ & Radix Angelicae Sinensis & Improves dysmenorrhea and irregular menstruation, shows anti-inflammatory activity \\
\hline Chishao $^{5}$ & Paeoniae Rubra Radix & Reduces fevers, induces sterility, and treats burns \\
\hline Dilong ${ }^{6}$ & Pheretima & Improves delirium (due to high fever), headache, acute conjunctivitis, hemiplegia \\
\hline Chuanxiong ${ }^{7}$ & Chuanxiong Rhizoma & $\begin{array}{l}\text { Anti-cerebral ischemia activity, anti-myocardial ischemia activity, protects vascular function, } \\
\text { antithrombotic activity, antihypertensive, anti-atherosclerotic, anti-inflammatory activity, anticancer } \\
\text { activity, antioxidant activity, liver protection }\end{array}$ \\
\hline Taoren 8,9 & Persicae Semen & $\begin{array}{l}\text { Anticoagulant, promotes blood circulation, removes blood stasis, relieves constipation, removes blood } \\
\text { stasis }\end{array}$ \\
\hline Honghua9 & Carthami Flos & Improves blood circulation, stimulates menstrual discharge, removes blood stasis, relieves pain \\
\hline
\end{tabular}

Table S5 Possible side/adverse effects of components of Bu Yang Huan Wu Tang

\begin{tabular}{|c|c|c|}
\hline English name & Official name & Possible adverse effects \\
\hline Huanqi $^{10}$ & Astragali Radix & Diarrhea, mild gastrointestinal effects, may affect blood sugar levels and blood pressure \\
\hline Danggui" $^{\prime \prime}$ & Radix Angelicae Sinensis & $\begin{array}{l}\text { Abdominal pain, amenorrhea, diarrhea, dystonia, gastrointestinal hemorrhage, hematemesis, irregular } \\
\text { menstruation, nausea, edema peripheral, pruritus, rash, somnolence, tongue spasm, urticaria, vaginal } \\
\text { hemorrhage }\end{array}$ \\
\hline Chishao $^{12}$ & Paeoniae Rubra Radix & $\begin{array}{l}\text { Blistering of mouth and throat, diarrhea, dizziness, fainting, gastroenteritis, hematuria, nausea, } \\
\text { salivation, stomach pain, vomiting }\end{array}$ \\
\hline Dilong $^{13}$ & Pheretima & Dizziness, fever, perspiration, photophobia, tearing, rummy nose, excess salivation, nausea \\
\hline Chuanxiong ${ }^{14-16}$ & Chuanxiong Rhizoma & Allergic reaction, vomiting, vertigo, nausea \\
\hline Taoren $^{17}$ & Persicae Semen & $\begin{array}{l}\text { Nausea, vomiting, irritation of the gastrointestinal tract, headache, dizziness, weakness, blurred vision, } \\
\text { increased heart rate, incontinence of the urine or stool, dilation of pupils }\end{array}$ \\
\hline Honghua ${ }^{17,18}$ & Carthami Flos & Bleeding, nausea, headache, dilated, pupils, increase in intraocular pressure, anaphylactic reaction \\
\hline
\end{tabular}




\section{References}

1. No authors listed. Astragalus membranaceus: monograph. Altern Med Rev. 2003;8(1):72-77.

2. Fu J, Wang Z, Huang L, et al. Review of the botanical characteristics, phytochemistry, and pharmacology of Astragalus membranaceus (Huangqi). Phytother Res. 2014;28(9):1275-1283.

3. Wu YC, Hsieh CL. Pharmacological effects of Radix Angelica sinensis (Danggui) on cerebral infarction. Chin Med. 2011;6:32.

4. Bain D. Pharmacological and biochemical action of Angelica sinensis (Dong Quai): natural product with therapeutic potential. Int J Recent Res Life Sci. 2015;2(3):8-23.

5. Lin MY, Chiang SY, Li YZ, et al. Anti-tumor effect of Radix Paeoniae Rubra extract on mice bladder tumors using intravesical therapy. Oncol Lett. 2016;12(2):904-910.

6. Chang HM, But PPH. Pharmacology and Applications of Chinese Materia Medica. Singapore: World Scientific Publishing Co. Ptd. Ltd; 1986-1987.

7. Chen Z, Zhang C, Gao F, et al. A systematic review on the rhizome of Ligusticum chuanxiong Hort. (Chuanxiong). Food Chem Toxicol. 2018;119:309-325.

8. Yang C, Li X, Rong J. Amygdalin isolated from Semen Persicae (Tao Ren) extracts induces the expression of follistatin in HepG2 and C2C12 cell lines. Chin Med. 2014;9:23.

9. Liu L, Duan JA, Tang Y, Guo J, Yang N, Ma H, Shi X. Taoren-Honghua herb pair and its main components promoting blood circulation through influencing on hemorheology, plasma coagulation and platelet aggregation. J Ethnopharmacol. 2012;139(2):381-387.
10. Astragalus. Maryland: National Center for Complementary and Integrative Health [updated September 2016]. Available from: https://nccih. nih.gov/health/astragalus. Accessed August 15, 2018.

11. European Medicines Agency. Assessment report on Angelica sinensis (Oliv.) Diels radix. [Updated July 9, 2013]. Available from: http://www. ema.europa.eu/docs/en_GB/document_library/Herbal_-_HMPC_assessment_report/2013/11/WC500155549.pdf. Accessed August 15, 2018.

12. European Medicines Agency. Assessment report on Paeonia lactiflora Pall. and Paeonia veitchii Lynch, radix (Paeoniae radix rubra). Available from: http://www.ema.europa.eu/docs/en_GB/document_library/ Herbal_-_HMPC_assessment_report/2016/02/WC500202014.pdf. Accessed August 15, 2018.

13. Marcus A. Foundations for Integrative Musculoskeletal Medicine: An East-West Approach. Berkeley (CA): North Atlantic Books; 2005.

14. Li X. Chinese Materia Medica: Combinations and Applications. 1st ed. Potters Bar: Donica Publishing; 2002.

15. Yang J, Huang H, Zhu LJ, Chen YH. World century compendium to TCM. Volume 3. Introduction to Chinese Materia Medica. Available from: https://books.google.com.tw/books?id=EH_FCwAAQBAJ\&pg $=\mathrm{PA} 324 \& \mathrm{dq}=$ Chuanxiong + Rhizoma+overdose $\& \mathrm{hl}=\mathrm{zh}-\mathrm{TW} \& \mathrm{sa}=\mathrm{X} \& \mathrm{v}$ ed=0ahUKEwjsoNSqiuDcAhUUO3AKHR25A6wQ6AEILTAB\#v=o nepage\&q\&f=true. Accessed August 15, 2018.

16. Jennes F, Flaws B. Herb Toxicities \& Drug Interactions: A Formula Approach. Boulder (CO): Blue Poppy Press; 2004.

17. Bensky D, Clavey S, Stöger E. Chinese Herbal Medicine: Materia Medica. 3rd ed. Seattle: Eastland Press; 2004.

18. Chen JK, Chen TT. Chinese Medical Herbology and Pharmacology. City of Industry (CA): Art of Medicine Press; 2004. 


\section{Publish your work in this journal}

Clinical Epidemiology is an international, peer-reviewed, open access, online journal focusing on disease and drug epidemiology, identification of risk factors and screening procedures to develop optimal preventative initiatives and programs. Specific topics include: diagnosis, prognosis, treatment, screening, prevention, risk factor modification,

Submit your manuscript here: https://www.dovepress.com/clinical-epidemiology-journal systematic reviews, risk and safety of medical interventions, epidemiology and biostatistical methods, and evaluation of guidelines, translational medicine, health policies and economic evaluations. The manuscript management system is completely online and includes a very quick and fair peer-review system, which is all easy to use. 